\title{
ISOGEOMETRIC ANALYSIS FOR VEHICLE MODEL IN DYNAMIC EXPLICIT METHOD
}

\author{
Y. YOKOYAMA ${ }^{1}$, H. SUGIYAMA ${ }^{2}$ AND S. OKAZAWA ${ }^{3}$ \\ ${ }^{1}$ Yuta Yokoyama \\ Department of Mechanical Engineering, University of Yamanashi, 4-3-11 Takeda, Kofu, Japan \\ g18dts03@yamanashi.ac.jp \\ ${ }^{2}$ Hirofumi Sugiyama \\ Department of Mechanical Engineering, University of Yamanashi, 4-3-11 Takeda, Kofu, Japan \\ hirofumis@yamanashi.ac.jp \\ ${ }^{3}$ Shigenobu Okazawa \\ Department of Mechanical Engineering, University of Yamanashi, 4-3-11 Takeda, Kofu, Japan \\ sokazawa@yamanashi.ac.jp
}

Key words: Isogeometric, Vehicle Crash Analysis, Dynamic Explicit, Automobile Component

\begin{abstract}
In Isogeometric Analysis, CAD format models can be used for analysis by using BSpline/NURBS, which is used for drawing shapes in CAD, as a shape function of the finite element method. This method can significantly reduce the time required to generate a mesh, which used to take much time. It is necessary to verify the performance of Isogeometric Analysis in the dynamic explicit method for application to automobile analysis. In this study, automobile structural parts were modeled by Isogeometric Analysis method and structural analysis was performed by the dynamic explicit method for evaluation.
\end{abstract}

\section{INTRODUCTION}

CAE (Computer-Aided Engineering), which performs simulations on a computer in product development and design, had become common. In FEM (Finite Element Method), the calculation is performed by discretizing the geometry data in CAD (Computer-Aided Design) format with a polygon mesh. The time spent on this mesh generation accounts for a large proportion of the entire analysis. In recent years, the models used in the analysis have become larger and larger, and the increase in time spent on mesh generation had become a problem. For example, in automobiles, it is said that it takes several months to generate a mesh for one automobile model.

IGA (Isogeometric Analysis) proposed by T.J.R. Hughes et al. is aimed at integrating CAE and CAD, and enables accurate geometry created by CAD to be handled directly by CAE [1-3]. In IGA, the NURBS basis function, which is generally used to generate curves and curved surfaces in CAD, is used as the shape function of the finite element method. With this method, it is not necessary to generate a mesh that occupies most of the time in the entire analysis, and it is possible to significantly reduce the cost. Furthermore, in FEM, an accurate and smooth model created by CAD must be approximated 
by polygon meshing for use in analysis, and there is a problem that the accuracy of the input model is reduced. In IGA, higher-order basis functions can be used, and analysis can be performed while retaining the shape created by CAD, so it is possible to avoid a decrease in analysis accuracy due to geometry error.

At the moment, the application of IGA to structural analysis has been actively studied [4,5]. Studies on analysis methods using trimmed curved surfaces [6-9] and the connection of Multi-NURBS patches have also been reported $[10,11]$. In the fluid analysis, cases where it is applied to the analysis of blood vessel flow such as aorta and heart valves, and cases where it is applied to fluid-structure coupling are also reported [12]. There is also an example of IGA performed by the explicit method by D.J. Benson, and its application to dynamic problems is also being promoted [13]. In recent years, IGA has been implemented in LS-DYNA, a commercial software that is a dynamic explicit solver [14-19]. However, there are not many reports on the study of deformation behavior such as buckling in dynamic phenomena and element performance such as mesh dependence, and there are few examples of application to actual structures. In the future, in order to expand the scope of application of IGA to dynamic phenomena such as automobile collision analysis, it will be necessary to evaluate the element performance by the explicit method.

In this study, we focus on the element performance of this IGA in the dynamic explicit method. In order to clarify that the behavior of deformation and buckling under dynamic phenomena such as collision can be predicted with high accuracy, IGA was applied to automobile members, and analysis was performed by the dynamic explicit method.
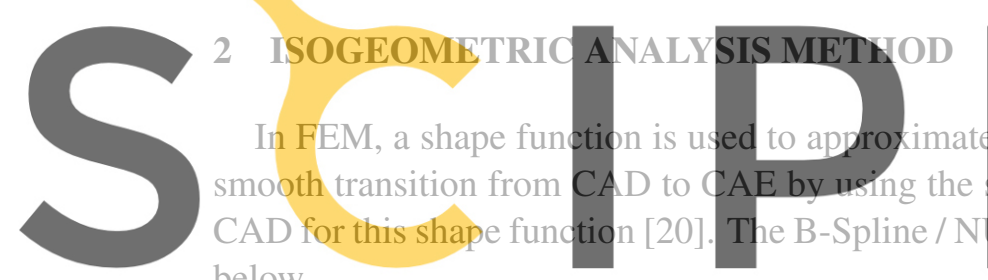
below.

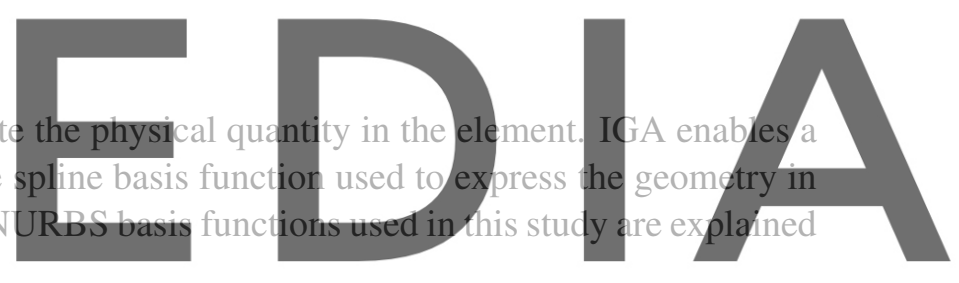

\section{Register for free at https_Lwwwyssipedia.com to download the version without the watermark}

The B-Spline curve determines the shape of the model based on the knot vector, which is a singie increasing sequence, and the coordinates of the control points. The B-Spline basis function is recursively defined by the following Cox-de Boor recurrence formula.

$$
\begin{gathered}
N_{i, 0}(\xi)= \begin{cases}1 & \text { if } \xi_{i} \leq \xi<\xi_{i+1} \\
0 & \text { otherwise. }\end{cases} \\
N_{i, p}(\xi)=\frac{\xi-\xi_{i}}{\xi_{i+p}-\xi} N_{i, p-1}(\xi)+\frac{\xi_{i+p+1}-\xi}{\xi_{i+p+1}-\xi_{i+1}} N_{i+1, p-1}(\xi)
\end{gathered}
$$

Here, $\xi_{i}$ is the $i$ th knot vector and $p$ is the order. The knot vector is a parameter required to determine the B-spline basis function, and is a sequence of monotonically increasing numbers expressed by the formula $N+P+1(N$ : number of control points $P$ : degree of polynomial). The knot vector in one dimension is $\xi=\left\{\xi_{1}, \xi_{2}, \cdots, \xi_{n}+p+1\right\}$. The subregions separated by this knot vector are defined as 
elements.

\subsection{NURBS BASIS FUNCTION}

NURBS is an abbreviation for Non-Uniform Rational B-Spline, which is an extension of the above B-Spline. NURBS was developed to draw some geometric shapes such as perfect circles that cannot be drawn with B-Spline curves. By considering the weight of the basis function, it is possible to control the curve passing near the control point and draw a perfect circle. The weight given to each control point has no absolute meaning, and the relative ratio between the individual control points affects the curve. The NURBS basis functions in three dimensions are shown below.

$$
R_{i, j, k}^{p, q, r}(\xi, \eta, \zeta)=\frac{N_{i, p}(\xi) M_{j, q}(\eta) L_{k, r}(\zeta) w_{i, j, k}}{\sum_{\hat{i}=1}^{n} \sum_{\hat{j}=1}^{m} \sum_{\hat{k}=1}^{l} N_{\hat{i}, p}(\xi) M_{\hat{j}, q}(\eta) L_{\hat{k}, r}(\zeta) w_{\hat{i}, \hat{j}, \hat{k}}}
$$

Where $N_{i, p}(\xi), M_{j, q}(\eta), L_{k, r}(\zeta)$ are B-spline basis functions in each parameter coordinate direction, $w_{i, j, k}$ are their weights, $i, j, k$ are the control point numbers, and $p, q, r$ are the orders of the B-Spline function.

The concept of basis functions in uniform knot vectors and non-uniform knot vectors is shown in Figure 1 and 2.
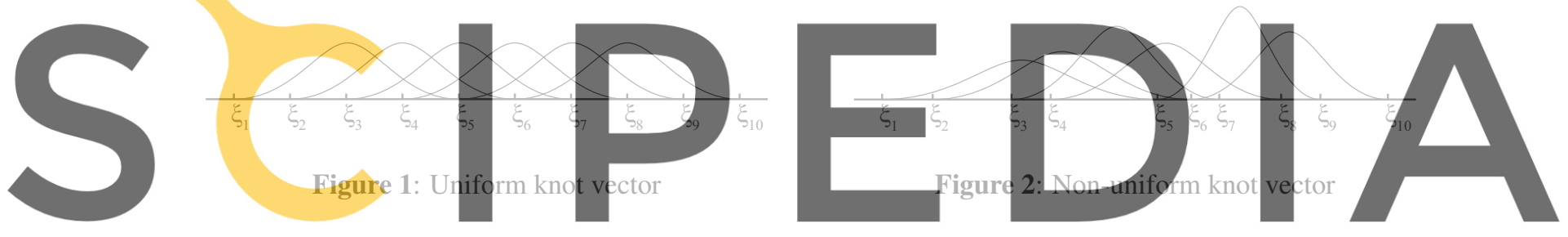

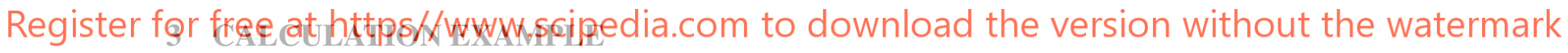

The trailing arm is one of the suspension parts of automobiles. In this chapter, IGA is used to analyze the three-point bending of the trailing arm. The results obtained by this analysis are compared with the results of the trailing arm static pressing test and FEM.

In the static pressing test, a trailing arm made of 370 class unplated hot-rolled steel sheet is used, and the center of the member is compressed vertically by an indenter at a velocity of $50 \mathrm{~mm} / \mathrm{min}$. The plate thickness is $2.3 \mathrm{~mm}$, the distance between the member supports is $474 \mathrm{~mm}$, and the shape of the indenter is a cylinder with a diameter of $17 \mathrm{~mm}$. The test conditions are shown in Figure 3 . The trailing arm is an elasto-plastic body, with mass density is $7860 \mathrm{~kg}$, Young's modulus is $205.8 \mathrm{Gpa}$, and poisson's ratio is 0.3 , and the true stress and the plastic curve are shown in Figure 4.

The Force-Stroke (FS) curves obtained from the test are used as reference solutions to compare and evaluate the simulation results.

The simulation model of FEM is the first-order element, the element size is $5 \mathrm{~mm}$, and the full integration is used. The IGA simulation model used a third-order NURBS patch, with a pitch spacing of $5 \mathrm{~mm}$ 


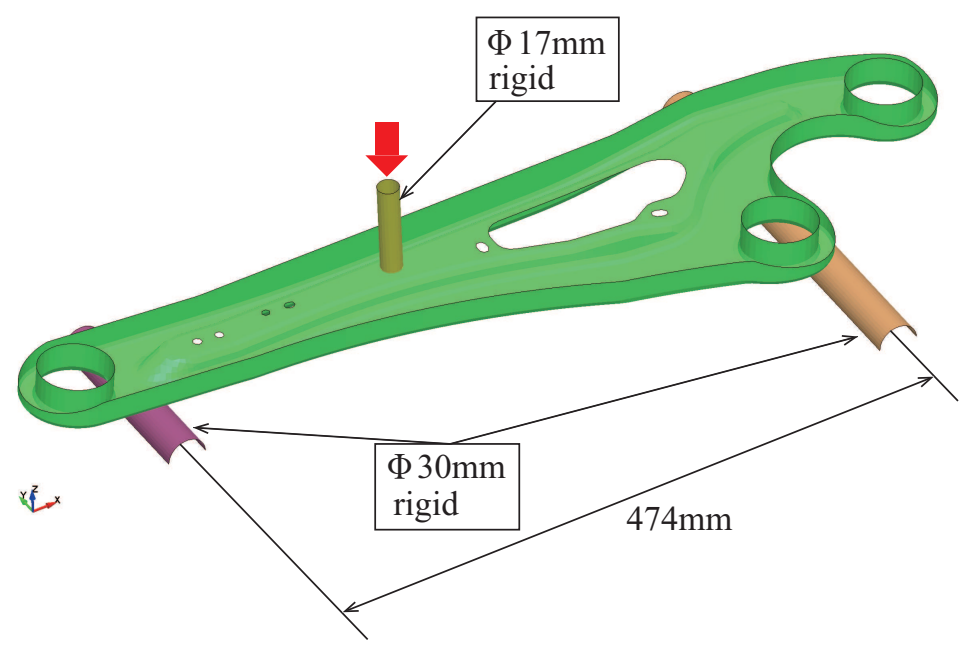

Figure 3: Schematic of three-point bending test of trailing arm
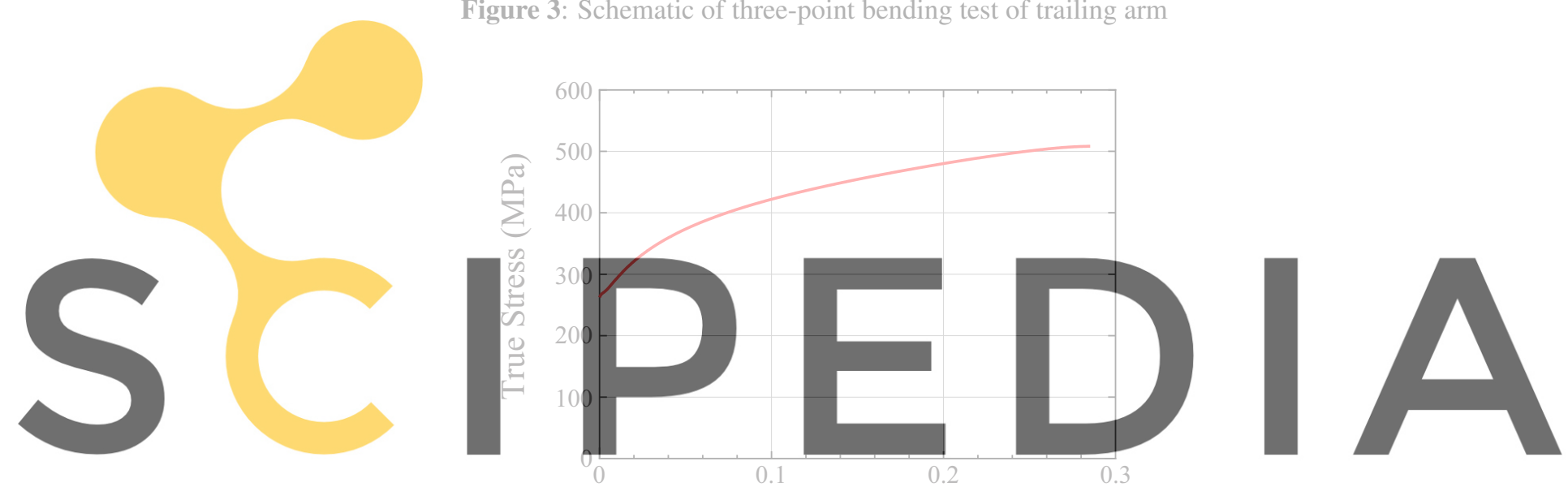

Register for free at https//www.scipedia.com Plagtiowhipliad the version without the watermark

Figure 4: True stress and plastic strain curve of Trailing arm

and an in-plane integration point of $3 \times 3$. In the simulation, the indenter velocity is $5000 \mathrm{~mm} / \mathrm{min}$. The indenter and support are rigid bodies.

The appearance of the IGA model is shown in Figure 5. The black line in the figure is the boundary of the subregion separated by the knot vector. In IGA, this subregion is an element in FEM, and integral calculation is performed in this region. The blue line of the model is the boundary of the NURBS patch. The model is created by multiple NURBS patches, and the small holes on the model are represented by trimming. NURBS patches are performed by sharing control points. Element boundaries separated by knot vectors in NURBS patches maintain $p-1$ continuity, but patch boundary continuity drops to $C^{0}$ when joined by node sharing.

The FS curve obtained from the test and simulation is shown in Figure 6. In the simulation, the FS curve similar to that in the test was obtained qualitatively. The results showed that the peak load was lower than that in the test. The trailing arm used in the test includes plate thickness variation and work 


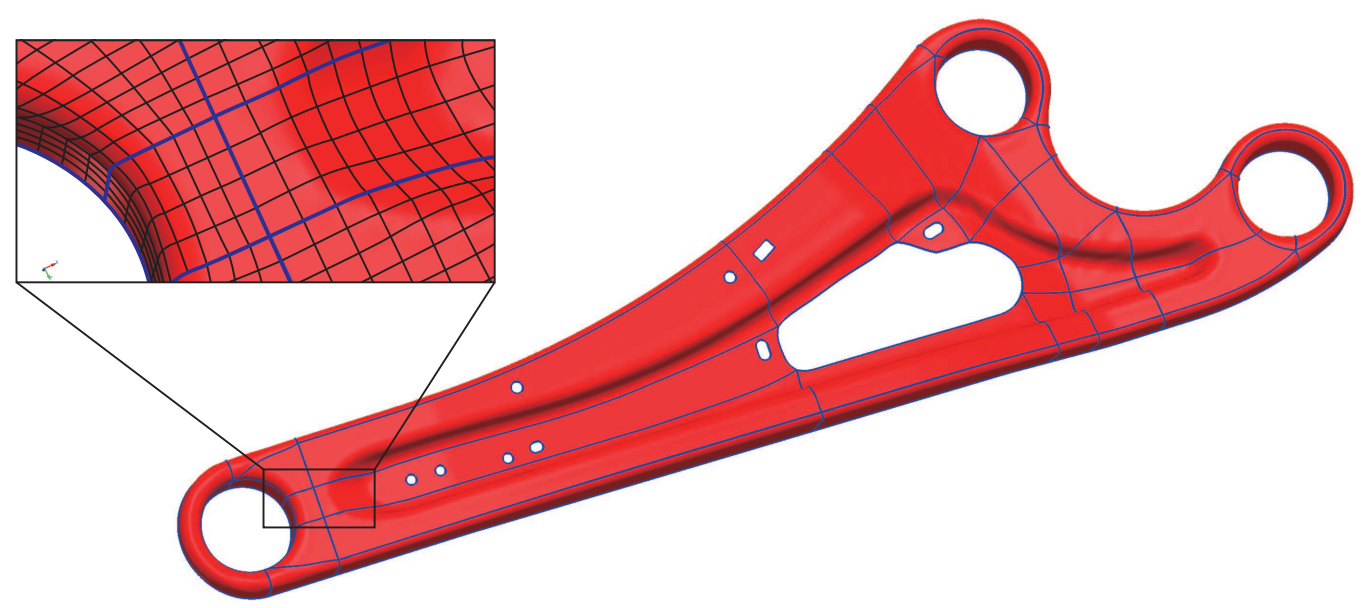

Figure 5: Trailing arm NURBS model

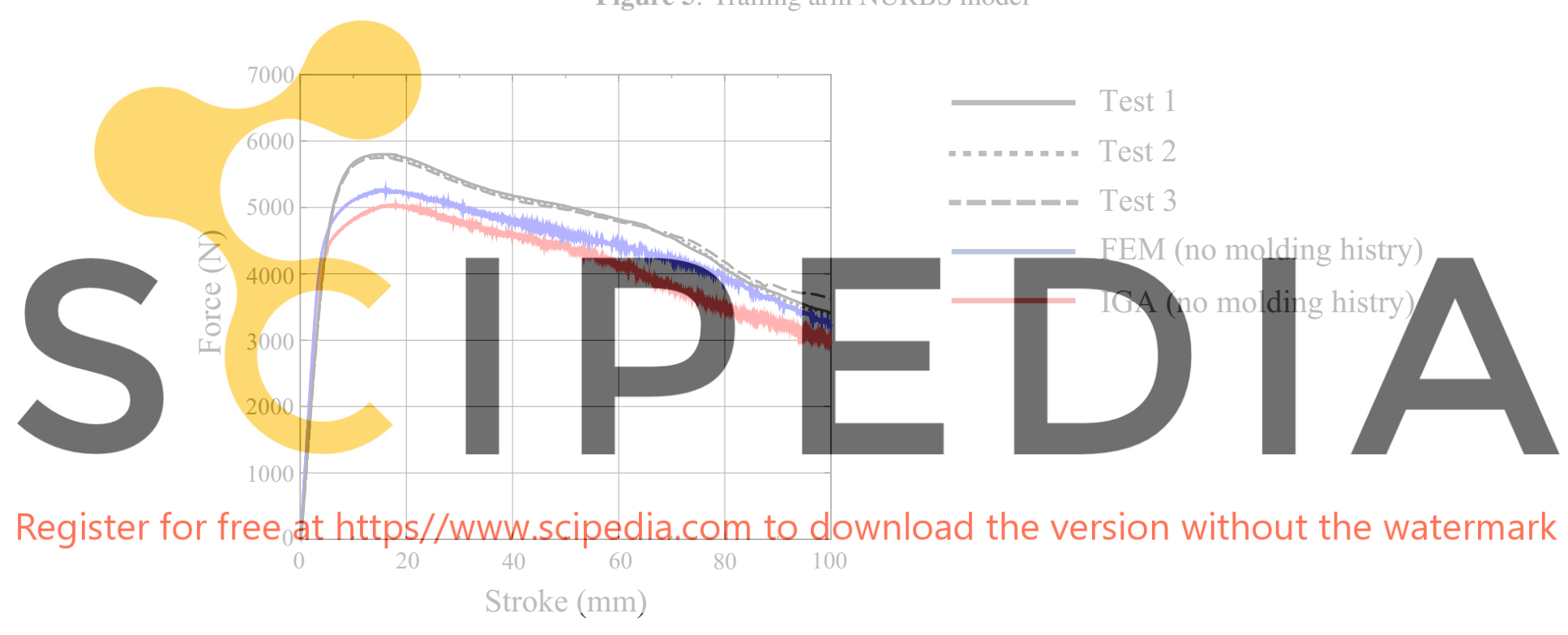

Figure 6: Force-Stroke Curve

hardening due to molding. However, the molding history is not set in the simulation model. Therefore, there was a difference in the peak value of the FS curve. When the molding history is set, the peak of the load rises and approaches the test value. The IGA curve had a slightly lower peak value than the FEM, however, a similar curve was obtained.

Figure 7 shows the deformation of the trailing arm model and the contour diagram of the Von-Mises stress. In this analysis, the IGA model obtained a stress distribution similar to that of FEM. There was a difference in deformation in the lower part of the center of the model, but this is the part where the deformation is not constant even in the test. In addition, there were some discontinuous stress distributions at the patch boundaries of the IGA model. This is thought to be because the continuity of the model decreased at the patch boundary. In this analysis, the element size was made the same for FEM and IGA, 


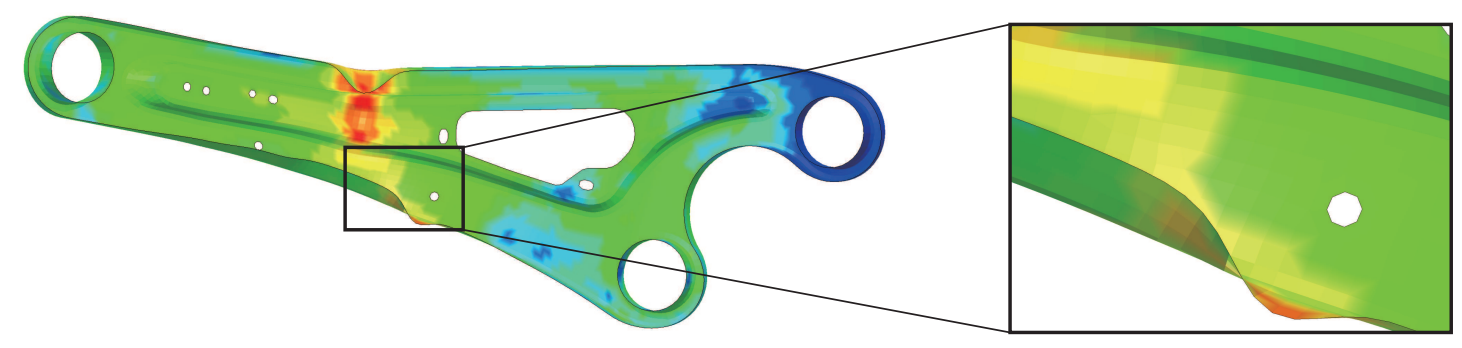

FEM

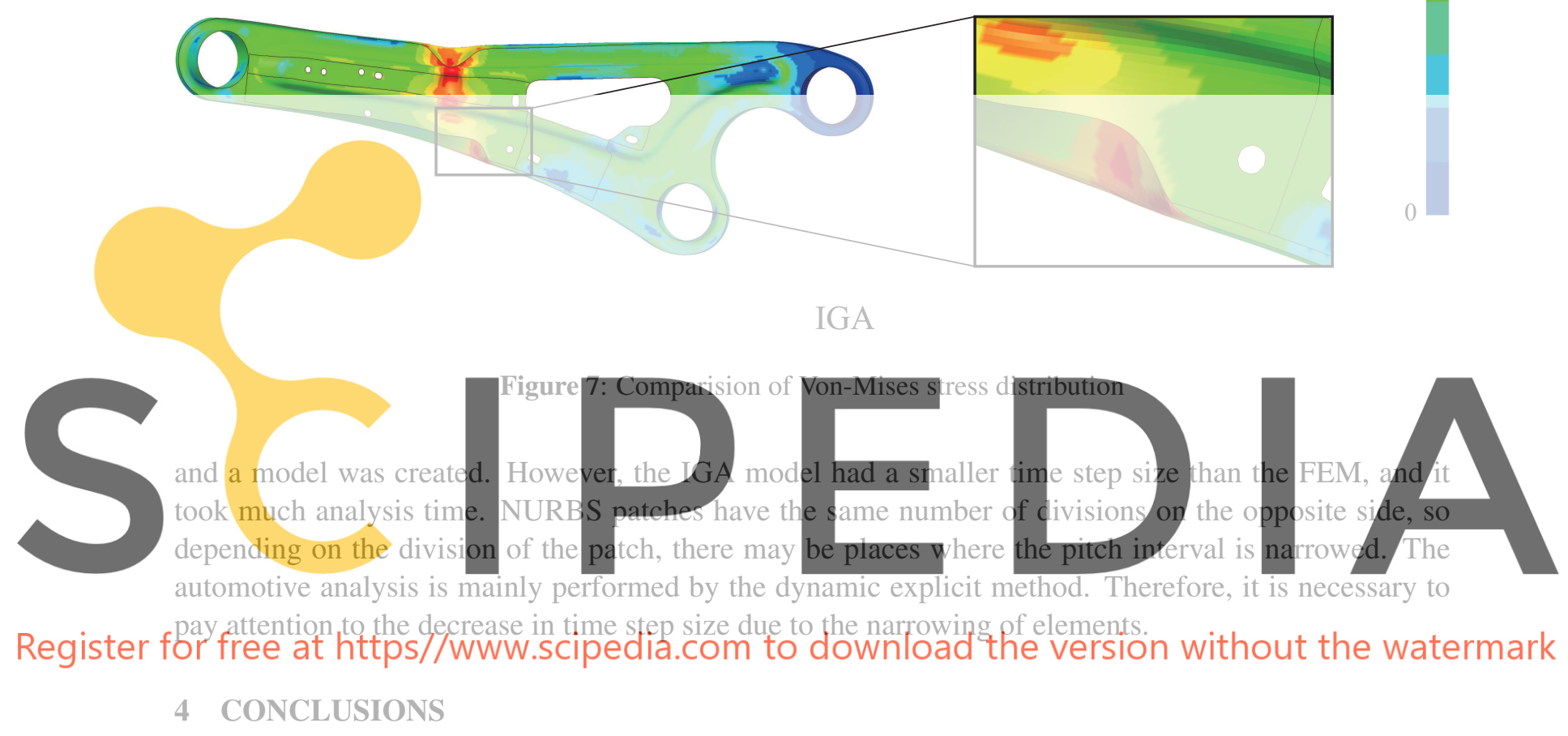

- In the simulation of the compression test using the trailing arm, which is a structural member of an automobile, FS curves qualitatively match the test values, and they also match well with the FEM.

- The distribution of Von-Mises stress in the IGA model was in good agreement with FEM. However, there was a phenomenon that the stress distribution was discontinuous at the boundary of the NURBS patch that was continuous with $C^{0}$, and there was a slight difference from the FEM result in some places.

- When creating a model by joining multiple NURBS patches using node sharing, the interval between control points may be locally reduced depending on how the patches are divided, and the time step size may become extremely small. Also, note that stress discontinuities may occur at the patch boundaries. 


\section{REFERENCES}

[1] Hughes, T.J.R., Cottrell, J.A., Bazilevs, Y., Isogeometric analysis:CAD, finite elements, NURBS, exact geometry and mesh refinemen, Comput. Methods Appl. Mech. Engrg., 194, (2005), pp. 41354195 .

[2] Cottrell, J.A., Reali, A., Bazilevs, Y., Hughes, T.J.R., Isogeometric analysis of structural vibrations, Comput. Methods Appl. Mech. Engrg., 195, (2006), pp. 5257-5296.

[3] Cottrell, J.A., Hughes, T.J.R., Bazilevs, Y., Isogeometric Analysis:Toward Integration of CAD and FEA, WILEY, (2009).

[4] Kiendl, J., Bletzinger, K.U., Linhard, J., Wüchner, R., Isogeometric shell analysis with KirchhoffLove elements, Comput. Methods Appl. Mech. Engrg., 198, (2009), pp. 3902-3914.

[5] Cottrell, J.A., Hughes, T.J.R., Reali, A., Studies of refinement and continuity in isogeometric structural analysis, Comput. Methods Appl. Mech. Engrg., 196, (2007), pp. 4160-4183.

[6] Kim, H.J., Seo, Y.D., Youn, S.K., Isogeometric analysis for trimmed CAD surface, Comput. Methods Appl. Mech. Engrg., 198, (2009), pp. 2982-2995.

[7] Sevilla, R., Fernández-Méndez, S., Numerical integration over 2D NURBS-shaped domains with applications to NURBS-enhanced FEM, Finite Elements in Anaysis ad Design, 47, (2011), pp. $1209-1220$.

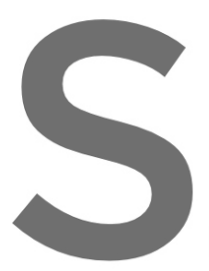

[8] Schmidt, R., Wüic Comput. Methods

[9] Nagy, A.P., Benson, Methods Appl. Mech. Engre

[10] Guo, Y., Rúess, M.,
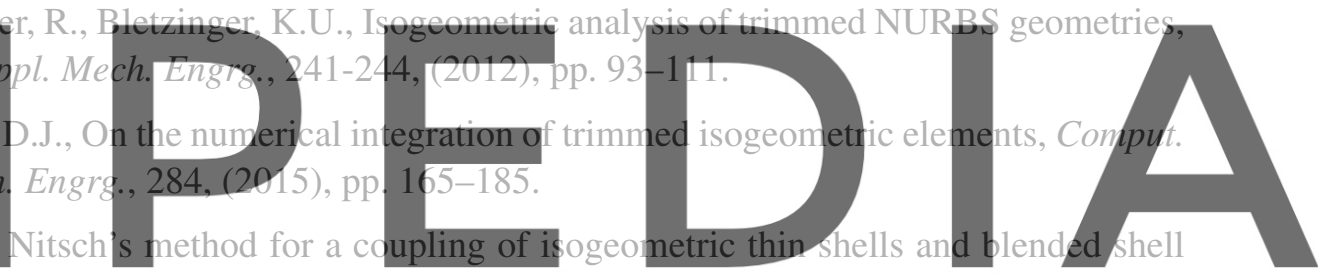

structures, Comput. Methods Appl. Mech. Engrg., 284, (2015), pp. 881-905.

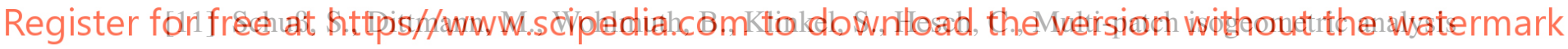
for kirchhoff-Love shell elements, Comput. Methods Appl. Mech. Engrg., 349, (2019), pp. 91-116.

[12] Miorganti, S., Auricchio, F., Benson, D.J., Gambarin, F.I., Hartmann, S., Hughes, T.J.R., Reaii, A., Patient-specific isogeometric structural analysis of aortic valve closure, Comput. Methods Appl. Mech. Engrg., 284, (2015), pp. 508-520.

[13] Benson, D., Bhalsod, D., Hartmann, S., Ho, P., Li, L., Li, W., Nagy, A., Yeh, I., Isogeometric Analysis in LS-DYNA: Using CAD-Geometry for Numerical Simulation, 10th European LS-DYNA Users Conference, Wurzburg, Germany, May, (2015).

[14] Hartmann, S., Benson, D.J., Lorenz, D., About Isogeometic Analysis and the New NURBS-Based Finite Element in LS-DYNA, 8th European LS-DYNA Users Conference, Strasbourg, France, May, (2011).

[15] Hartmann, S., Introduction to Isogeometric Elements in LS-DYNA, 2011 Developer Forum, Stuttgart-Filderstadt, Germany, October, (2011).

[16] Hartmann, S., Current Status of Isogeometic Analysis in LS-DYNA, 2013 Developer Forum, 
Stuttgart-Filderstadt, Germany, September, (2013)

[17] Hartmann, S., Benson, D.J., Mass scaling and stable time step estimates for isogeometric analysis, Int. J. Numer. Meth. Engrg, 102, (2015), pp. 671-687.

[18] Chen, Y., Lin, S.P., Faruque, O., Alanoly, J., El-Essawi, M., and Baskaran, R., Current Status of LS-DYNA Iso-geometric Analysis in Crash Simulation, 14th International LS-DYNA Users Conference, Dearborn, Michigan, June, (2016).

[19] Hartmann, S., Nagy, A.P., Benson, D.J., Advances in IGA for Sheet Metal Forming Applications, 11th European LS-DYNA Users Conference, Salzburg, Austria, May, (2017).

[20] Piegl, L., Tiller, W., The NURBS Book, Springer, (1997).
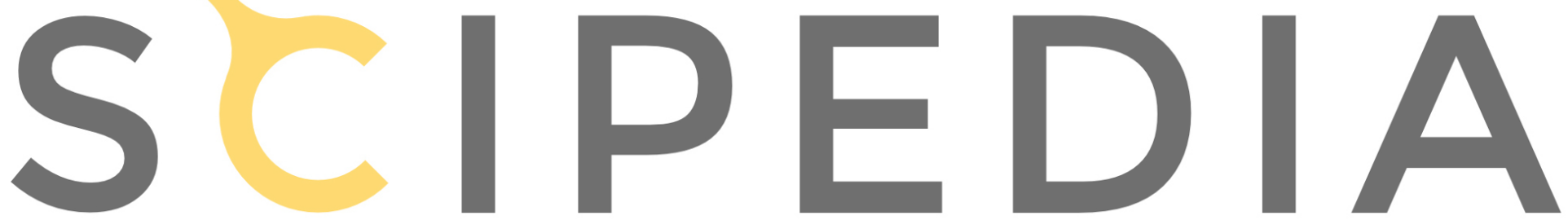

Register for free at https//www.scipedia.com to download the version without the watermark 